\title{
Fertility-Sparing Surgery for Patients with Endometrioid Stromal Sarcoma of Ovary: A Case Report with a Review of the Literature
}

\section{Bo Ra Park}

Department of Obstetrics and Gynecology, Soonchunhyang University Seoul Hospital, Seoul, Korea

\begin{abstract}
Endometrioid stromal sarcoma (ESS) of ovary is very rare disease. These mesenchymal neoplasms occur most commonly in the uterus and occasionally originate from extra-uterine sites, such as the ovary. It is often associated with endometriosis of the ovary. Here we present this rare case to emphasize on the clinicopathologic features and fertility-sparing surgery outcome of ovarian ESS in patients with endometriosis.
\end{abstract}

Keywords: Endometrioid stromal tumor; Ovarian neoplasms; Fertility preservation

\section{INTRODUCTION}

Primary endometrioid stromal sarcomas (ESS) of the ovary are rare mesenchymal tumors. It occurs in women over wide age range of 11 years to 76 years accounting for only $0.2 \%$ of all uterine malignancies and for $10 \%-15 \%$ of primary uterine sarcomas [1]. ESS is originated from cells that resemble those of the endometrial stroma during the proliferative phase of the menstrual cycle [2]. When uterine in location, ESS is easy to diagnose because of their characteristic histology and patterns of invasion. However, when they occur at extrauterine sites, they produce nongynecologic signs and symptoms. The majority of extrauterine ESS arises from endometriosis, suggesting that most of these tumors originate by malignant transformation of the stromal component of endometriosis [3]. ESS arising from endometriosis is commonly well differentiated and considered as an indolent tumor with good prognosis.

We recently encountered a case of ESS with endometriosis in pre-menopause woman. To provide an insight into the sites of occurrence, clinicopathologic features and fertility-sparing surgery outcome of ESS, we report a case of ESS with endometriosis with literature review.

\section{CASE REPORT}

A 25-year-old, unmarried woman came to gynecology outpatient department with the complaint of both ovarian mass which was noticed January, 2013, by accidently. Her menstrual cycles were regular but were associated with dysmenorrhoea. Routine papanicolaou smear showed reactive cellular changes with inflammation. Ultrasound examination and computed tomography (CT) revealed thin-walled slightly high attenuated (about 30 Hounsfield unit) lesion measuring $60 \times 31 \mathrm{~mm}$ in right adnexa (Fig. 1). There was no abnormal lymphadenopathies or fluid collection in abdomen and pelvis. And left adnexa had $40 \times 29 \mathrm{~mm}$ monoloculated diffuse hypoechoic mass (Fig. 2). On physical examination, the patient was generally well, weighed $57.6 \mathrm{~kg}$ and not pale. Hematological examination was within normal range. The patient underwent laparoscopic right ovarian pseudocystectomy with removal of left ovarian mass with adhesiolysis on March 7, 2013. At operation, right ovary had 6-cm sized pseudocyst include chocolate like material and adhered to uterus posterior wall. Left ovary had $4-\mathrm{cm}$ sized yellow and fragile solid mass adhered to uterus posterior wall (Fig. 3). Tumor arising from left ovary consists of multiple fragments of partly brownish and partly yellowish, friable soft tis- 


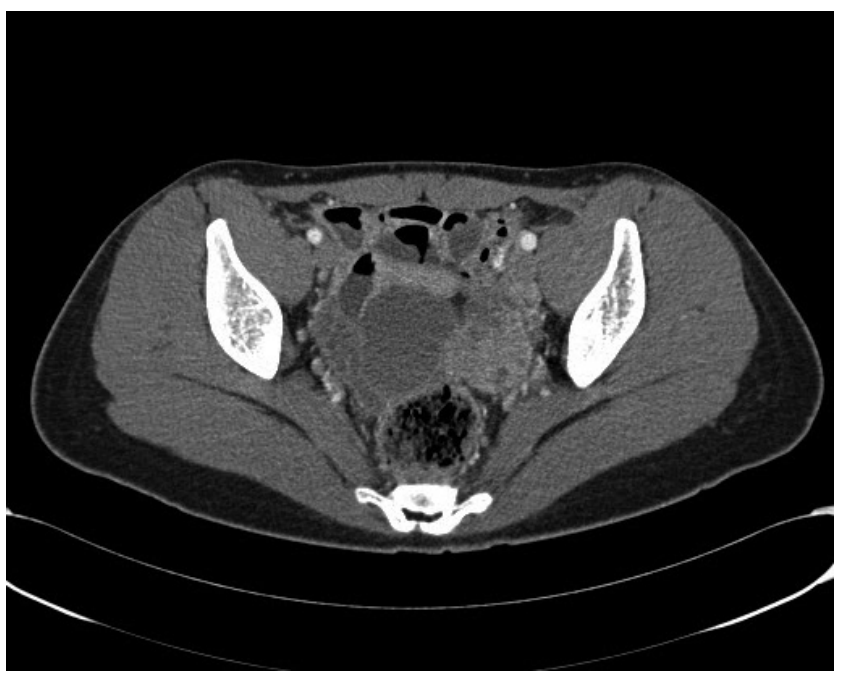

Fig. 1. 52×34-mm sized thin-walled slightly high attenuated labout 30 Hounsfield unit) mass in right adnexa at enhanced computed tomography.

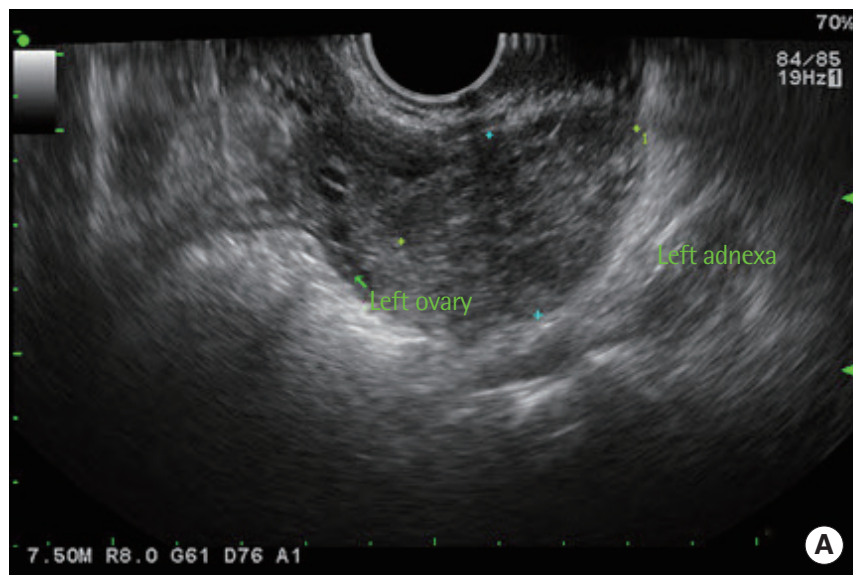

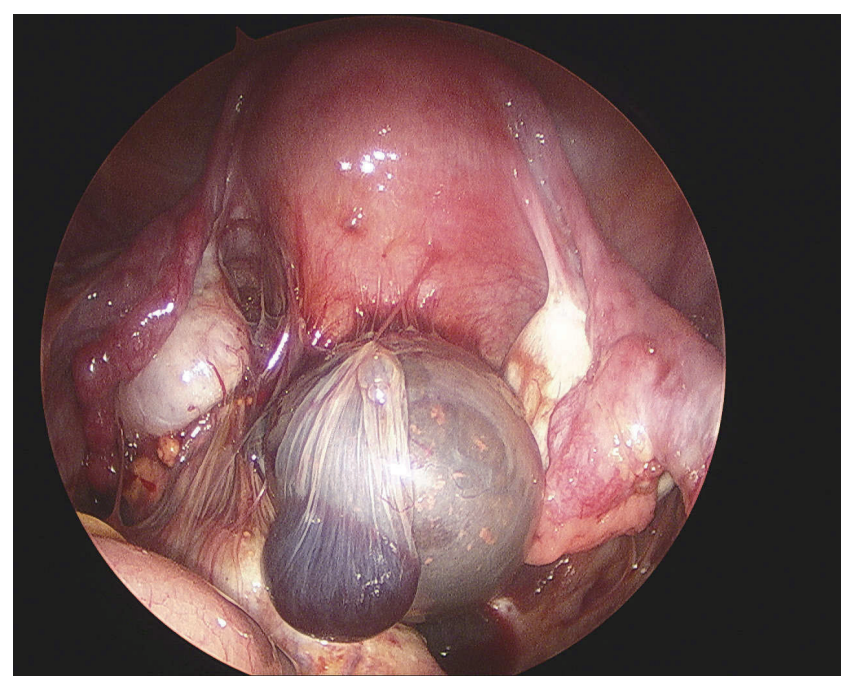

Fig. 3. Right ovary had 6-cm sized pseudocyst include chocolate-like material and adhered to uterus posterior wall. Left ovary had 4-cm sized yellow and fragile mass. This mass adhered to uterus posterior wall.

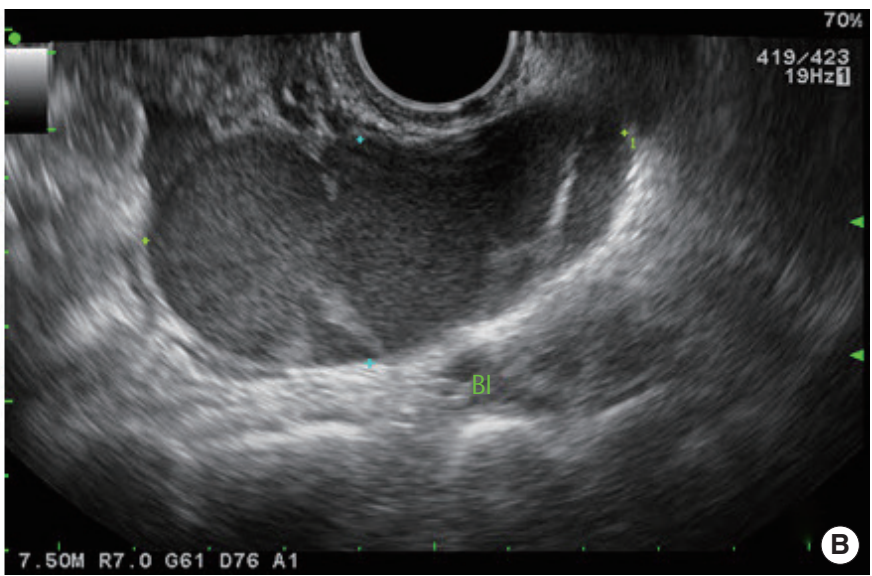

Fig. 2. Transverse ultrasonography image shows 40×29-mm monoloculated diffuse hypoechoic mass in left adnexa (A) and 60×31-mm sized hypoechoic multiloculated mass in right adnexa (B).

sue. Specimen retrieval bag was used for contained removal of adnexal masses because of the concern regarding spread of malignant cells. Final pathology was consistent with left ovarian ESS with endometriosis. Grossly, the left ovarian tumor consists of multiple fragments of irregularly shaped brown and yellowish fragile soft tissue. Microscopically, the tumor shows diffuse proliferation of small round cells with a few small arterioles. A transition between the tumor and endometriosis is seen. The tumor is composed of relatively small cells with oval to fusiform nuclei and inconspicuous nucleoli (Fig. 4). The tumor was strongly and diffusely positive for estrogen receptor, progesterone receptor, and desmin. CD10 and Ki-67 (less than 5\%) were focally positive.
However, calretinin, D2-40, CD34, and inhibin-alpha were all negative (Fig. 5).

When histological results confirmed the diagnosis of ESS, we performed positron emission tomography (PET)-CT and rescheduled for laparoscopic left oophorectomy for preserving fertility. Torso PET-CT showed $11 \times 11 \times 10$-mm sized hypermetabolic nodule in left ovary, cystic mass in right adnexa without FDG uptakes. No evidence of lymphadenopathy in entire pelvis, abdomen, and mediastinum. In March 26, 2013, laparoscopic left oophorectomy, endometrial biopsy, adhesiolysis, and washing cytology was done. No evidence of tumor was found in the endometrium. The result of peritoneal cytology was negative for malignant cells, but a 

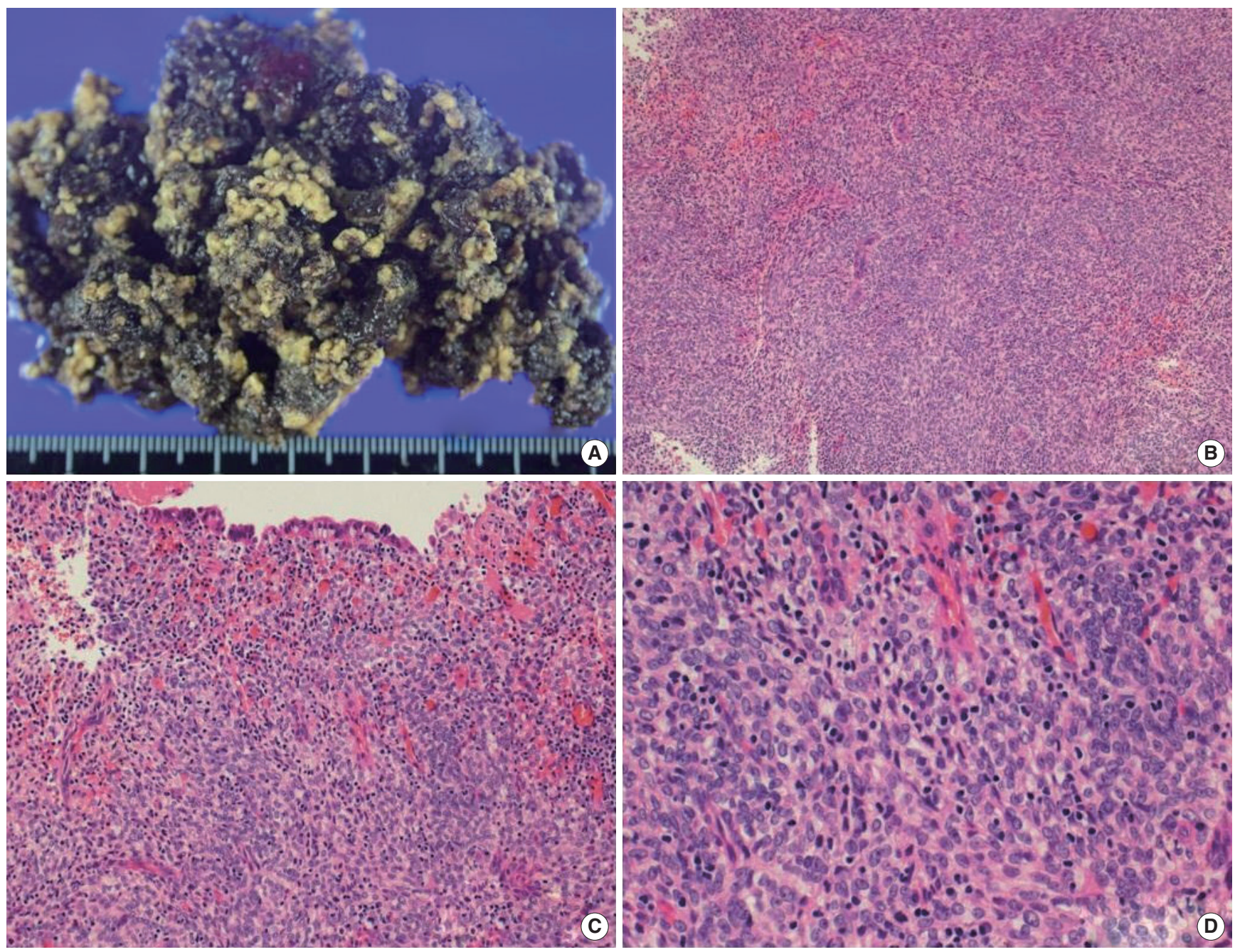

Fig. 4. (A) Grossly, the tumor consists of multiple fragments of irregularly shaped brown and yellowish fragile soft tissue. (B) Microscopically, the tumor shows diffuse proliferation of small round cells with a few small arterioles $(H \& E, \times 40)$. (C) A transition between the tumor and endometriosis is seen (H\&E, $\times 100)$. (D) The tumor is composed of relatively small cells with oval to fusiform nuclei and inconspicuous nucleoli (H\&E, $\times 400)$.

few clusters of reactive mesothelial cells are seen. We have not encountered any recurrence in the study at this time following conservative treatment.

\section{DISCUSSION}

ESSs are characterized by infiltrative growth of cells resembling normal proliferative-phase endometrial stroma. Endometriosis is commonly identified adjacent to the neoplastic tissue, so it suggests that there would be correlation between ESS and endometriosis. Young et al. [4] found that endometriosis presented in 11 cases among 23 ESS cases. According to Baiocchi et al. [5], 29 of 45 cases of extrauterine ESSs qualified as arising from endometriosis by Sampson's criteria [6]. Above Sampson's three criteria was (1) clear examples of endometriosis in close proximity to tumor, (2) no other primary site of malignancy, and (3) histological appearance compatible with origin from endometriosis. Baiocchi et al. [5] found approximately half of the patients were nulliparous. It would be explained by that $30 \%$ to $40 \%$ of patients with endometriosis are infertile. However, Ober and Black [7] suggest another possibility for the origin of this tumor is metaplasia from the ovarian surface mesothelium or from the subcoelomic mesenchyme.

Tumors are usually solid, but others are solid and cystic and sometimes cystic. The surfaces of solid areas are yellow-white and some cases have foci of hemorrhage or necrosis. Our case also has yellow and fragile mass that identified ESS. Microscopic features 

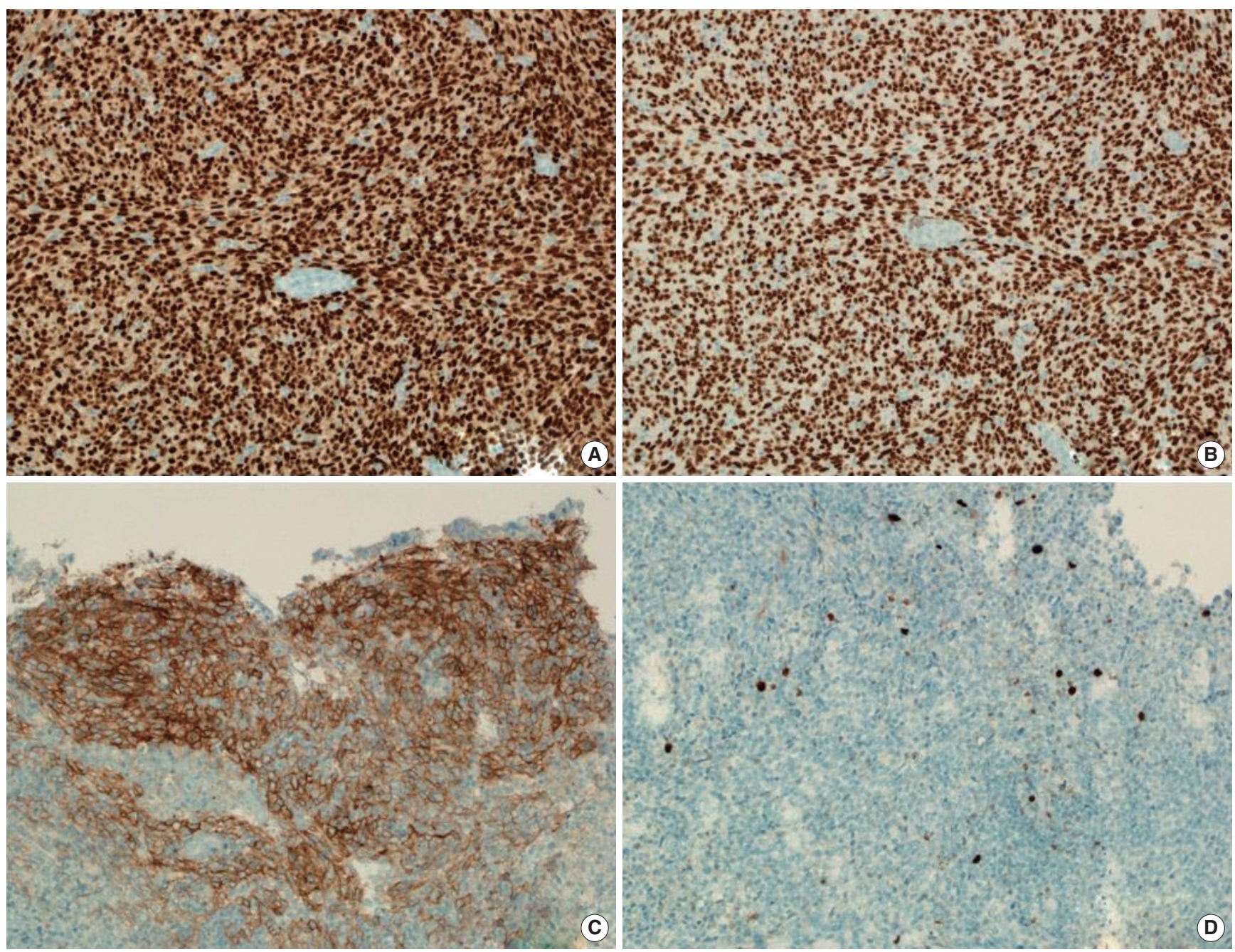

Fig. 5. Immunostaining of the tumor. The tumor cells are positive for estrogen receptor (A), progesterone receptor (B), and CD10 (C) and low level of MIB-1 expression (D).

of ESSs are typically composed of sheets of uniform cells, which are usually round or oval, but occasionally have a spindle shape and resemble the stromal cells of normal proliferative endometrium. Numerous small blood vessels resembling the spiral arterioles of late secretory endometrium are present. The neoplastic cells usually cluster around these vessels. The nuclei are oval to round and nucleoli are present but not prominent. The cytoplasm is pale and scanty and the cell borders are not clearly recognizable. There is sometimes fibromatous areas. Foam cells and vascular invasion often present in the tumors [4].

Immunohistochemically, tumors express vimentin, muscle markers like desmin, muscle-specific actin and $\alpha$-smooth muscle actin, and progesterone receptors [8]. Recent studies have described the unique expression of CD10 in endometrial stromal cells as well as in ESS, which was proven diagnostically useful in the distinction of ESS from smooth muscle neoplasms like lowgrade leiomyosarcoma [9]. Other study suggests CD 34 might be helpful in diagnosing extrauterine extraovarian ESS [10].

Tumors with less than 10 mitosis per 10 high power fields (HPF) are classified as low grade and those with 10 or more as high grade. Chang et al. [11] reported that neither mitotic index nor cytologic atypia in primary extrauterine endometrial stromal neoplasm were predictive of tumor recurrence or death from tumor. In their study, $77 \%$ of patients whose tumors had a mitotic index of less than 10 per HPF had one or more recurrences and 30\% died from their neoplasm.

Low-grade ESS has an indolent clinical course with a tendency for late recurrence. In stage I low-grade ESS, 5- and 10-year survival rates have been estimated to be $98 \%$ and $89 \%$, respectively.

Primary therapy is complete resection of neoplasm. Thus, if the 
patient is perimenopausal or postmenopausal, hysterectomy with bilateral salpingoophorectomy is recommended. The effect of chemotherapy in both low grade and high grade endometrioid stromal sarcomas is unclear. Cyclophosphamide, adriamycin, vincristine, methotrexate is used and some case have complete remission, but follow-up is short [4]. Also there is no clear evidence about effectiveness of radiotherapy and progesterone therapy. The risk of recurrence is as high as $50 \%$ along with interval for recurrence and a median interval of about three years from hysterectomy to relapse [12]. Because performing fertility-sparing surgery for lowgrade ESS is rare, and because the long-term follow-up results are still lacking, it is not possible to draw any definitive conclusion about its safety.

In conclusion, ovarian ESS has an indolent clinical course, and is associated with a favorable prognosis; ovary-sparing procedures might be considered in younger patient. However, no standard treatment strategy has yet been established. Future investigation should include more study participants and focus on the response to treatment and prognosis.

\section{ACKNOWLEDGMENTS}

This work was supported in part by the Soonchunhyang University Research Fund.

\section{REFERENCES}

1. Back JA, Choi MG, Ju UC, Kang WD, Kim SM. A case of advanced-stage endometrial stromal sarcoma of the ovary arising from endometriosis. Obstet Gynecol Sci 2016;59:323-7.

2. Diesing D, Cordes T, Finas D, Loning M, Mayer K, Diedrich K, et al. Endometrial stromal sarcomas: a retrospective analysis of 11 patients. Anticancer Res 2006;26:655-61.

3. Masand RP. Unusual presentations of gynecologic tumors: primary, extrauterine, low-grade endometrioid stromal sarcoma. Arch Pathol Lab Med 2018;142:536-41.

4. Young RH, Prat J, Scully RE. Endometrioid stromal sarcomas of the ovary: a clinicopathologic analysis of 23 cases. Cancer 1984;53:1143-55.

5. Baiocchi G, Kavanagh JJ, Wharton JT. Endometrioid stromal sarcomas arising from ovarian and extraovarian endometriosis: report of two cases and review of the literature. Gynecol Oncol 1990;36:147-51.

6. Sampson JA. Endometrial carcinoma of the ovary, arising in endometrial tissue in that organ. Arch Surg 1925;10:1-72.

7. Ober WB, Black MB. Neoplasms of the subcoelomic mesenchyme; report of two cases. AMA Arch Pathol 1955;59:698-705.

8. Fukunaga M, Ishihara A, Ushigome S. Extrauterine low-grade endometrial stromal sarcoma: report of three cases. Pathol Int 1998;48:297-302.

9. Corpa MV, Serafini EP, Bacchi CE. Low-grade endometrial stromal sarcoma presenting as vaginal nodule. Ann Diagn Pathol 2004;8:295-8.

10. Kim L, Choi SJ, Park IS, Han JY, Kim JM, Chu YC, et al. Endometrial stromal sarcoma of the small bowel. Ann Diagn Pathol 2008;12:128-33.

11. Chang KL, Crabtree GS, Lim-Tan SK, Kempson RL, Hendrickson MR. Primary extrauterine endometrial stromal neoplasms: a clinicopathologic study of 20 cases and a review of the literature. Int J Gynecol Pathol 1993;12:282-96.

12. Berceanu S, Patrascu A, Berceanu C, Tica AA, Badulescu A. Endometrial stromal sarcoma: clinico-pathological report of four cases and review of the literature. Rom J Morphol Embryol 2008;49:251-5. 